\title{
Dimensiones mesiodistales de las coronas de la dentición permanente en niños escolares de Lima
}

\author{
Artículo ORIGINAL
}

\section{Mesiodistal crown diameters of permanent dentition in schoolchildren from Lima}

\begin{abstract}
Resumen
El propósito de este estudio fue conocer el tamańo de los diámetros mesiodistales de los dientes permanentes en una población escolar de la ciudad de Lima, para analizar las discrepancias entre dientes homólogos, dimorfismo sexual de dientes permanentes, así como el grado de variabilidad de las medidas. Metodología: la investigación se realizó en 400 escolares de raza mestiza (200 hombres y 200 mujeres) de 10 a 12 ańos de edad, de tres centros educativos de los distritos de La Victoria y Barrios Altos, de la ciudad de Lima. El procedimiento se realizó directo en boca empleando un calibrador Vernier. Resultados y conclusiones: se encontró que en el maxilar superior la $1 \mathrm{M}$ presentó el mayor diámetro mesiodistal seguido en orden descendente por la 2M, IC, C, 1PM, 2PM, IL, en el maxilar inferior fue mayor la $1 \mathrm{M}$ seguido en orden descendente por la 2M, 2PM, 1PM, C, IL, C. La diferencia de promedios entre dientes homólogos del lado derecho e izquierdo fue muy pequeńa en ambos géneros, no mostrando significancia estadística. Los diámetros mesiodistales del sexo masculino fueron significativamente mayores que los del femenino, excepto en el caso de la segunda premolar superior, las mayores diferencias se presentaron en la primera y segunda molar en el maxilar superior, y en el canino y primera molar en el caso del maxilar inferior. Los dientes que presentaron mayor variabilidad de tamańo en el maxilar superior fueron los incisivos laterales en ambos géneros, y en el maxilar inferior, los incisivos centrales en los hombres y segundos premolares en las mujeres, los cuales se identifican como las piezas con mayor potencial para provocar alteraciones intra e inter arcadas. Los promedios hallados en el presente estudio son mayores que los encontrados por otros autores.
\end{abstract}

Palabras clave: Dimensiones mesiodistales; Tamańo dental; Dentición permanente.

\section{Abstract}

The purpose of this study was to determine the size of mesiodistal diameters of permanent teeth in a school population of the city of Lima, to analyze the differences between homologous teeth, sexual dimorphism of permanent teeth as well as the degree of variability of the measurements. Methodology: The study was conducted on 400 students of mestizos (200 men and 200 women) 10 to 12 years, from three schools in the districts of la Victoria and Barrios Altos, Lima city. The procedure was performed directly in the mouth using a Vernier caliper. Results and conclusions: it was found that $1 \mathrm{M}$ in the maxillary had the greatest mesiodistal diameter followed in descending order by 2M, IC, C, 1PM, $2 \mathrm{PM}$, IL, in the mandible the $1 \mathrm{M}$ was higher followed in descending order by the $2 \mathrm{M}, 2 \mathrm{PM}, 1 \mathrm{PM}$, C, IL, C. The mean difference between homologous teeth of the right and left side was very small in both genders, showing no statistical significance. The mesiodistal diameters of males were significantly higher than those of female, except in the case of the maxillary second premolar, the largest differences occurred in the first and second molar in the maxillary and the canine, first and second molars in the mandible. Teeth that showed greater variability in dimensions were the lateral incisors in the maxilla in both genders and the central incisors in males and second premolars in females in the mandible, which were identified as teeth with the greater potential to cause intra and inter arcades alterations. Averages found in the present study were higher than those found by other authors.

Keywords: Mesiodistal dimensions; Tooth size; Permanent dentition.

\section{Introducción}

El tamaño de los dientes es uno de los determinantes para obtener una oclusión normal, los dientes maxilares y mandibulares deben guardar una determinada proporción para obtener una correcta oclusión, además de estar en armonía con el tamaño de arcada para que pueda darse una alineación correc$\mathrm{ta}^{1,2}$.
El diagnóstico ortodóncico estaría incompleto sin un cuidadoso análisis de las relaciones de los tamaños coronales mesiodistales, ya que éstas dimensiones son factores decisivos para: a) el desarrollo de irregularidades oclusales y faciales, b) posibles discrepancias en la intercuspidación, durante y después del tratamiento ortodóncico y c) el aislamiento de dientes discrepantes, en maloclusiones menores $^{12}$.
Martha Pineda-Mejía ${ }^{1}$, Marieta PetkovaGueorguieva²

1. Departamento Académico de Estomatología Rehabilitadora, Facultad Odontología de la Universidad Nacional Mayor de San Marco, Perú.

2. Departamento Académico de Ciencias Básicas, Facultad Odontología de la Universidad Nacional Mayor de San Marcos, Perú.

\section{Correspondencia:}

Mg. Martha Pineda-Mejía

Facultad Odontologia, Universidad Nacional Mayor de San Marcos, Av. Germán Amezaga s/n. Lima 1, Perú.

Correo electrónico: mpinedam@unmsm.edu.pe

Coautora:

Petkova-Gueorguieva:

mpetkovag@unmsm.edu.pe

Fecha de recepción: 15-12-13

Fecha de aceptación: 27-06-14
La variación en tamaño de los dientes está influenciada por factores genéticos y factores ambientales ${ }^{8}$. Varios estudios han reportado la variación del tamaño de los dientes entre y dentro de diferentes grupos raciales, Kenee y col. ${ }^{14}$, informaron diferencias raciales en los tamaños de los dientes entre reclutas navales libres de caries caucásicos y negros americanos. Turner y Richardson ${ }^{13}$ también 
observaron diferencias significativas en la anchura dentaria mesiodistal en las poblaciones kenianas e irlandeses. En otro estudio relacionado, Bisha$\mathrm{ra}^{15}$ comparó las dimensiones coronarias mesiodistales y buco-linguales de dientes permanentes en tres poblaciones de Egipto, México y el Estados Unidos, los resultados de este estudio mostraron diferencias estadísticamente significativas en los valores hallados entre las tres poblaciones. Aparte de las diferencias raciales, los otros factores asociados con la variabilidad del tamaño de los dientes son: el genero ${ }^{16,17}$, medio ambiente ${ }^{19}$, factores hereditarios, diferencias bilaterales y cambios seculares. Variaciones del tamaño de los dientes se han reportado entre diversos grupos étnicos poblaciones como americanos caucásicos del norte, negros, mongoloides, dominicanos, mexicanos, egipcios, nigerianos, británico, etc. Variaciones raciales también han sido observadas en los esquimales, bosquimanos, japoneses, aborígenes australianos y las razas bantúes de África.

Como se ha observado en varias investigaciones, el tamańo de los dientes es de gran importancia no sólo para indicar las diferentes actividades relacionadas con la oclusión o para determinar la frecuencia de las anomalías dento-dentales aplicados al tratamiento de ortodoncia, sino también para establecer el dimorfismo sexual $^{11}$. Esto, por lo tanto, tendrá una gran importancia en la medicina forense. Debido a esto, el conocimiento de las dimensiones coronarias para la identificación del sexo cuando se encuentran esqueletos se vuelve relevante, especialmente cuando los parámetros anatómicos no son fiables para la identificación de un tema en particular. Esto disminuiría obtener el número de individuos buscados a una probabilidad de $50 \%$, lo que, junto con la información obtenida a partir de un documento de registro clínico (hecho durante el tratamiento de rehabilitación para cada paciente), puede resultar en una forma más precisa de la identificación de la persona buscada.

Richardson y col. ${ }^{14}$, encontraron que los dientes de los hombres eran más grandes que aquellos de las mujeres para cada tipo de diente en ambas arcadas. Sanin y Savara ${ }^{12}$ reportaron diferencias en los patrones de los tamaños dentarios incluso entre casos de buena oclusión. Adeyemi ${ }^{3}$ encon- tró en su estudio que los anchos mesiodistales combinados eran mayores en hombres que en mujeres. Además, Moorrees $^{2}$ en su estudio de 184 niños blancos norteamericanos reportó que los hombres tenían anchos mesiodistales más grandes que las mujeres.

En nuestro medio, hay muy poca información sobre los tamaños mesiodistales de los dientes de peruanos mestizos, muchos odontólogos generales y especialistas, adoptan auxiliares diagnósticos propios de otros grupos étnicos, principalmente caucásicos americanos (Bishara) ${ }^{6}$, (Richardson $)^{14}$, (Moorrees y col.) $)^{2}$.

Los objetivos del presente estudio fueron:

- Determinar las dimensiones coronarias mesiodistales de la dentición permanente en una población escolar de 10 a 12 ańos de edad de la ciudad de Lima - Perú.

- Comparar los promedios mesiodistales de dientes homólogos según genero

- Hacer un análisis de la variabilidad de las medidas mesiodistales por tipo de diente

- Determinar el dimorfismo sexual en cuanto a las dimensiones mesiodistales de los dientes permanentes

- Realizar un análisis comparativo con promedios encontrados en otros países

\section{Materiales y método}

El diseño corresponde a un estudio descriptivo, transversal. La muestra estuvo integrada por 400 niños escolares (200 hombres, 200 mujeres) de 10 a 12 años de edad, que estaban cursando el $5^{\circ}$ y $6^{\circ}$ grado de primaria, de los centros educativos 1110, 1050, 1028; de los distritos de La Victoria y Barrios Altos, de la ciudad de Lima, procedentes de padres peruanos y de raza mestiza. La selección de la muestra se hizo con los siguientes criterios:

1. Que los niños no presentaran desarmonías oclusales, alteraciones del crecimiento, anomalías congénitas o enfermedades generales severas con repercusiones cráneo facial.

2. Que presentaran una clase I molar sin apińamiento.

3. Que existiera normalidad en cuanto al número, tamaño y forma dentaria.
4. Que no existiese migraciones dentales por pérdidas prematuras de dientes temporales.

5. Que los dientes presentaran integridad anatómica. Si los dientes presentaban restauraciones, estas no debían afectar al tamaño mesiodistal del diente.

6. Que no hayan recibido tratamiento ortodóncico con o sin extracciones.

7. Que no estén en proceso de erupción.

Se obtuvo el consentimiento informado de los padres o responsables de los niños que integraron la muestra.

El procedimiento de medición de los diámetros coronarios se realizó directo en boca, para lo cual previamente a cada estudiante se le hacia la remoción de materia alba, tártaro y saliva de las caras proximales de los dientes, luego empleando un compás metálico biactivo de puntas aguzadas y paralelas entre sí, para los dientes anteriores y el compás de puntas anguladas para los dientes posteriores. Seleccionados los dientes se procedió a la medición colocando las puntas del compás biactivo paralelo a la superficie oclusal y vestibular, a nivel del punto de contacto de los dientes detectado deslizando las puntas del compás por las caras proximales por la zona de mayor anchura; la medida se inmovilizaba mediante un tornillo estabilizador y luego se procedió a su lectura exacta usando el calibrador de Vernier.

Para poder medir los anchos mesiodistales, algunos autores han optado por hacer la medición directamente en boca y otros, a través de modelos de estudio, no existiendo diferencias significativas de mediciones entre $\operatorname{ambos}^{4,7}$.

\section{Resultados}

Tabla.1

Promediosdelos diámetros mesiodistales dentarios en escolares de Lima

\begin{tabular}{ccc}
\hline $\begin{array}{c}\text { TIPO } \\
\text { DIENTE }\end{array}$ & $\begin{array}{c}\text { MAXILAR } \\
\text { SUPERIOR }\end{array}$ & $\begin{array}{c}\text { MAXILAR } \\
\text { INFERIOR }\end{array}$ \\
\hline IC & $08.84 \pm 0.52$ & $05.71 \pm 0.39$ \\
IL & $07.18 \pm 0.57$ & $06.33 \pm 0.43$ \\
C & $08.24 \pm 0.44$ & $07.21 \pm 0.41$ \\
1PM & $07.52 \pm 0.43$ & $07.38 \pm 0.45$ \\
2PM & $07.26 \pm 0.44$ & $07.44 \pm 0.48$ \\
1M & $11.10 \pm 0.56$ & $11.42 \pm 0.58$ \\
2M & $10.49 \pm 0.60$ & $11.02 \pm 0.60$ \\
\hline
\end{tabular}


Tabla 2. Comparación de los diámetros mesiodistales de dientes permanentes. Lima

\begin{tabular}{|c|c|c|c|c|c|c|c|c|c|}
\hline \multirow[b]{2}{*}{$\begin{array}{l}\text { MAXILAR } \\
\text { SUPERIOR }\end{array}$} & \multicolumn{4}{|c|}{ MASCULINO $(n=200)$} & \multicolumn{4}{|c|}{ FEMENINO $(n=200)$} & \multirow[b]{2}{*}{$t$} \\
\hline & MEDIA & DS & RANGO & CV & MEDIA & DS & RANGO & CV & \\
\hline 11 & 8.94 & 0.56 & $10.30-7.80$ & 6.27 & 8.72 & $0-50$ & $10.30-7.60$ & 5.74 & $p<0.01$ \\
\hline 21 & 8.97 & 0.54 & $10.20-7.80$ & 6.02 & 8.72 & 0.48 & $10.30-7.60$ & 5.50 & $p<0.01$ \\
\hline 12 & 7.32 & 0.58 & $8.90-5.90$ & 7.92 & 7.04 & 0.56 & $8.30-5.00$ & 7.95 & $p<0.01$ \\
\hline 22 & 7.29 & 0.57 & $9.00-5.90$ & 7.81 & 7.06 & 0.54 & $8.60-5.20$ & 7.65 & $p<0.01$ \\
\hline 13 & 8.40 & 0.44 & $9.50-7.30$ & 5.24 & 8.10 & 0.44 & $9.40-7.10$ & 5.43 & $p<0.01$ \\
\hline 23 & 8.34 & 0.44 & $9.30-7.30$ & 5.28 & 8.10 & 0.44 & $9.60-7.00$ & 5.43 & $p<0.01$ \\
\hline 14 & 7.66 & 0.45 & $8.80-6.60$ & 5.88 & 7.46 & 0.40 & $8.60-6.50$ & 5.36 & $p<0.01$ \\
\hline 24 & 7.55 & 0.45 & $8.30-6.10$ & 5.96 & 7.44 & 0.40 & $8.60-6.40$ & 5.38 & $p<0.05$ \\
\hline 15 & 7.30 & 0.45 & $8.50-5.90$ & 6.16 & 7.24 & 0.41 & $8.70-6.20$ & 5.66 & $p>0.05$ \\
\hline 25 & 7.22 & 0.48 & $8.60-6.10$ & 6.65 & 7.33 & 0.42 & $8.30-6.10$ & 5.73 & $p<0.05$ \\
\hline 16 & 11.34 & 0.60 & $12.60-9.10$ & 5.29 & 11.00 & 0.52 & $12.50-9.70$ & 4.73 & $p<0.01$ \\
\hline 26 & 11.31 & 0.55 & $12.90-9.40$ & 4.86 & 10.75 & 0.57 & $12.20-9.80$ & 5.30 & $p<0.01$ \\
\hline 17 & 10.66 & 0.69 & $12.80-9.10$ & 6.48 & 10.38 & 0.59 & $11.80-8.40$ & 5.69 & $p<0.01$ \\
\hline 27 & 10.54 & 0.59 & $12.20-9.50$ & 5.60 & 10.28 & 0.54 & $11.70-9.20$ & 5.25 & $p<0.01$ \\
\hline \multicolumn{10}{|l|}{ M. INFERIOR } \\
\hline 31 & 5.77 & 0.42 & $7.20-5.00$ & 7.28 & 5.59 & 0.34 & $6.60-4.90$ & 6.08 & $p<0.01$ \\
\hline 41 & 5.84 & 0.44 & $7.50-5.00$ & 7.53 & 5.64 & 0.33 & $6.50-4.90$ & 5.85 & $p<0.01$ \\
\hline 32 & 6.36 & 0.47 & $7.50-5.20$ & 7.39 & 6.22 & 0.39 & $7.50-5.20$ & 6.27 & $p<0.01$ \\
\hline 42 & 6.50 & 0.46 & $7.90-5.40$ & 7.08 & 6.25 & 0.39 & $7.40-5.30$ & 6.24 & $p<0.01$ \\
\hline 33 & 7.43 & 0.46 & $8.50-6.50$ & 6.19 & 7.00 & 0.38 & $8.30-5.60$ & 5.43 & $p<0.01$ \\
\hline 43 & 7.42 & 0.43 & $8.30-6.30$ & 5.80 & 7.00 & 0.38 & $8.20-6.00$ & 5.43 & $p<0.01$ \\
\hline 34 & 7.66 & 0.50 & $8.30-6.10$ & 6.53 & 7.22 & 0.41 & $8.20-6.10$ & 5.68 & $p<0.01$ \\
\hline 44 & 7.47 & 0.47 & $8.80-6.30$ & 6.29 & 7.19 & 0.41 & $8.40-6.10$ & 5.70 & $p<0.01$ \\
\hline 35 & 7.53 & 0.46 & $8.30-6.30$ & 6.11 & 7.30 & 0.48 & $8.70-6.20$ & 6.58 & $p<0.01$ \\
\hline 45 & 7.56 & 0.49 & $8.90-6.20$ & 6.48 & 7.36 & 0.48 & $8.70-6.20$ & 6.52 & $p<0.01$ \\
\hline 36 & 11.45 & 0.62 & $13.20-10.60$ & 5.41 & 11.27 & 0.53 & $12.90-10.20$ & 4.70 & $p<0.05$ \\
\hline 46 & 11.76 & 0.60 & $13.20-10.30$ & 5.10 & 11.20 & 0.55 & $12.90-9.50$ & 4.91 & $p<0.01$ \\
\hline 37 & 11.24 & 0.65 & $13.30-9.90$ & 5.78 & 10.83 & 0.57 & $12.60-9.30$ & 5.26 & $p<0.01$ \\
\hline 47 & 11.10 & 0.60 & $13.30-9.70$ & 5.41 & 10.89 & 0.57 & $12.50-9.60$ & 5.23 & $p<0.01$ \\
\hline
\end{tabular}

Tabla 3. Diámetros mesiodistales según género en escolares de Lima

\begin{tabular}{cccccccc}
\hline MAXILAR & \multicolumn{3}{c}{ MASCULINO } & \multicolumn{3}{c}{ FEMENINO } & \multirow{2}{*}{$\mathbf{t}$} \\
\cline { 2 - 7 } SUPERIOR & MEDIA & DS & CV & MEDIA & DS & CV & \\
\hline IC & 8.95 & 0.55 & 6.15 & 8.72 & 0.49 & 5.62 & $\mathrm{p}<0.01$ \\
IL & 7.30 & 0.58 & 7.95 & 7.05 & 0.55 & 7.80 & $\mathrm{p}<0.01$ \\
C & 8.37 & 0.44 & 5.26 & 8.10 & 0.44 & 5.43 & $\mathrm{p}<0.01$ \\
1PM & 7.59 & 0.45 & 5.93 & 7.45 & 0.40 & 5.37 & $\mathrm{p}<0.01$ \\
2PM & 7.23 & 0.46 & 6.36 & 7.28 & 0.42 & 5.77 & $\mathrm{p}>0.05$ \\
1M & 11.32 & 0.57 & 5.03 & 10.87 & 0.54 & 4.97 & $\mathrm{p}<0.01$ \\
2M & 10.64 & 0.64 & 6.02 & 10.33 & 0.56 & 5.42 & $\mathrm{p}<0.01$ \\
M. INFERIOR & & & & & & & \\
IC & 5.80 & 0.43 & 7.41 & 5.61 & 0.34 & 6.06 & $\mathrm{p}<0.01$ \\
IL & 6.43 & 0.46 & 7.15 & 6.23 & 0.39 & 6.26 & $\mathrm{p}<0.01$ \\
C & 7.42 & 0.44 & 5.93 & 7.00 & 0.38 & 5.43 & $\mathrm{p}<0.01$ \\
1PM & 7.55 & 0.48 & 6.36 & 7.21 & 0.41 & 5.69 & $\mathrm{p}<0.01$ \\
2PM & 7.54 & 0.47 & 6.23 & 7.33 & 0.48 & 6.55 & $\mathrm{p}<0.01$ \\
1M & 11.60 & 0.61 & 5.26 & 11.23 & 0.54 & 4.80 & $\mathrm{p}<0.01$ \\
2M & 11.17 & 0.62 & 5.55 & 10.86 & 0.57 & 5.25 & $\mathrm{p}<0.01$ \\
\hline
\end{tabular}

\section{Discusión}

Diámetros mesiodistales de la muestra estudiada

El análisis de los diámetros coronarios mesiodistales se hizo promediando los valores del lado derecho con el izquierdo, pues según los resultados, existe una gran simetría entre ambos lados, los promedios se muestran en la Tabla 1 , se encontró que en el maxilar superior la $1 \mathrm{M}$ presentó el mayor diámetro, seguido en orden descendente por la 2M, IC, C, 1PM, 2PM, siendo el IL el diente de menor diámetro en la arcada.

En el maxilar inferior la $1 \mathrm{M}$ presentó el mayor diámetro, seguido en orden descendente por la $2 \mathrm{M}, 2 \mathrm{PM}, 1 \mathrm{PM}, \mathrm{C}, \mathrm{IL}$ siendo el IC el diente de menor diámetro en la arcada.

El hecho de que las primeras molares superiores e inferiores sean los dientes más grandes y el incisivo lateral superior y el incisivo central inferior sean los dientes más pequeños de la boca, es un patrón que se repite en los hallazgos de muchos autores ${ }^{2,8,9}$.

\section{Comparación de dientes homólogos.}

Los valores promedios mesiodistales de los dientes correspondientes a ambos lados de la línea media fueron en general muy similares. Los resultados de la prueba t de Student, corroboraron lo anterior, ya que no se encontraron diferencias significativas en ninguno de los 14 pares de dientes evaluados, tanto 
Tabla 4. Comparación de diámetros mesiodistales

\begin{tabular}{ccccccccc}
\hline & \multicolumn{3}{c}{ MASCULINO } & \multicolumn{3}{c}{ FEMENINO } \\
\hline $\begin{array}{c}\text { MAXILAR } \\
\text { SUPERIOR }\end{array}$ & Lima & $\begin{array}{c}\text { USA } \\
\mathbf{( 2 )}\end{array}$ & $\begin{array}{c}\text { North } \\
\text { Indian } \\
\mathbf{( 5 )}\end{array}$ & $\begin{array}{c}\text { México } \\
\mathbf{( 8 )}\end{array}$ & Lima & $\begin{array}{c}\text { USA } \\
\mathbf{( 2 )}\end{array}$ & $\begin{array}{c}\text { North } \\
\text { Indian } \\
\mathbf{( 5 )}\end{array}$ & $\begin{array}{c}\text { México } \\
\mathbf{( 8 )}\end{array}$ \\
\hline IC & 8.95 & 8.78 & 9.05 & 8.50 & 8.72 & 8.40 & 8.62 & 8.32 \\
IL & 7.30 & 6.64 & 7.07 & 6.73 & 7.05 & 6.47 & 6.95 & 6.67 \\
C & 8.37 & 7.95 & 8.16 & 7.93 & 8.10 & 7.53 & 7.86 & 7.67 \\
1PM & 7.59 & 7.01 & 7.35 & 7.16 & 7.45 & 6.85 & 7.20 & 7.11 \\
2PM & 7.23 & 6.82 & 7.10 & 6.83 & 7.28 & 6.62 & 6.76 & 6.75 \\
1M & 11.32 & 10.81 & 10.35 & 10.25 & 10.87 & 10.52 & 10.03 & 10.07 \\
2M & 10.64 & 10.35 & 9.95 & 9.94 & 10.33 & 9.81 & 9.57 & 9.71 \\
M- INFERIOR & & & & & & & & \\
IC & 5.80 & 5.42 & 5.68 & 5.40 & 5.61 & 5.25 & 5.55 & 5.39 \\
IL & 6.43 & 5.95 & 6.31 & 6.03 & 6.23 & 5.78 & 5.98 & 5.86 \\
C & 7.42 & 6.95 & 7.26 & 6.94 & 7.00 & 6.47 & 6.88 & 6.54 \\
1PM & 7.55 & 7.07 & 7.42 & 7.18 & 7.21 & 6.87 & 7.02 & 7.00 \\
2PM & 7.54 & 7.29 & 7.55 & 7.17 & 7.33 & 7.02 & 7.17 & 7.09 \\
1M & 11.60 & 11.80 & 11.23 & 11.38 & 11.23 & 10.74 & 10.80 & 11.00 \\
2M & 11.17 & 10.76 & 10.33 & 10.38 & 10.86 & 10.34 & 10.01 & 10.07 \\
\hline & & & & & & & &
\end{tabular}
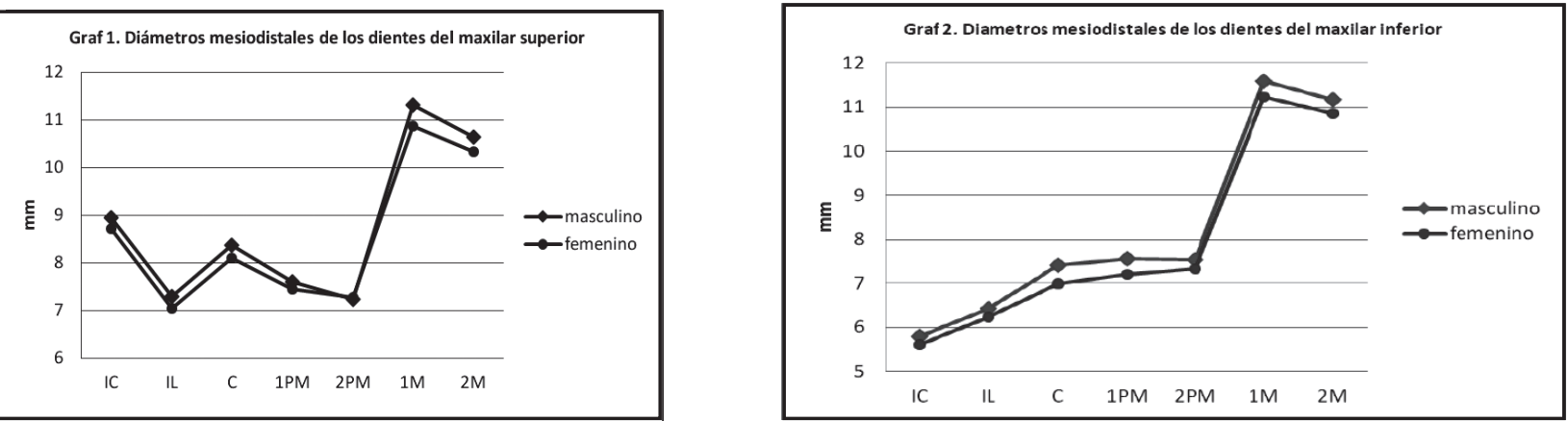

en hombres como en mujeres. En la Tabla 2 se presentan los promedios coronarios mesiodistales de cada uno de los 28 dientes medidos.

La diferencia promedio entre los dientes del lado derecho e izquierdo, fue de 0.11 para los varones y de 0.07 para las mujeres. En la mayoría de los pares comparados $(85.7 \%$ en hombres y $25 \%$ en las mujeres), resultaron ser ligeramente más grandes los dientes del lado derecho que sus homólogos izquierdos.

En los varones, los dientes del lado derecho que presentaron promedios mesiodistales ligeramente mayores que sus homólogos del lado izquierdo fueron: ILS, CS, 1PMS, 2PMS, IMS, 2MS, ICI, ILI, 2PMI, 2MI, por lo contrario los ICS, CI, 1PMI y 1MI del lado izquierdo fueron ligeramente mayores que sus homólogos derechos, sin embargo, estas diferencias no presentaron significancia estadística, para la prueba t, $p>0.05$. Las mayores diferencias se presentaron en las $2 \mathrm{MS}$ y $1 \mathrm{MI}$, y las menores diferencias en ILS, 1MS y CI.

En las mujeres, se observó una situación un poco diferente: siete tipos de dientes del lado derecho presentaron diámetros ligeramente mayores que sus homólogos del lado izquierdo en los casos de 1PMS, $1 \mathrm{MS}, 2 \mathrm{MS}$, ICI, ILI, 2PMI y $2 \mathrm{MI}$, por el contrario cuatro tipos de dientes del lado izquierdo fueron ligeramente mayores que sus homólogos del lado derecho ILS, 2PMS, 1PMI y $1 \mathrm{MI}$, estas diferencias no presentaron significancia estadística para la prueba $t, p>0.05$, y finalmente tres tipos de dientes presentaron iguales promedios en ambos lados: ICS, CS, CI.

Las diferencias fueron tan pequeñas que no son significativas clínicamente. Este hallazgo es consistente en estudios pre$\operatorname{vios}^{2,14,15}$, sin embargo, difieren de lo reportado por Ballard en 1944, quien estudió la simetría del tamaño dental mesiodistal, a ambos lados de la línea media en 500 casos, encontrando en el $90 \%$ de su muestra, una discrepancia $\geq 0.25 \mathrm{~mm}$. en la presente investigación, los valores del lado derecho fueron en su mayoría ligeramente más grandes que los del lado izquierdo (17 de 28 pares), coincidiendo con resultados de Khan ${ }^{9}$ y en contraposición a los resultados de Moorrees ${ }^{2}$ quien en una población americana y Kubodera ${ }^{8}$ en una población mexicana, encontraron que los dientes del lado izquierdo eran en su mayoría ligeramente mayores que los del lado derecho.

Estos resultados indican que, en ambos sexos, al medir tamaños dentarios en dentición permanente, se pueden utilizar solo las mediciones de un lado de la arcada (de- recho ó izquierdo), o la media entre el lado derecho y el izquierdo.

\section{Dimorfismo sexual}

$\mathrm{Al}$ analizar el dimorfismo sexual en cuanto al tamaño coronario de los dientes permanentes se encontró que los diámetros mesiodistales de los dientes del maxilar superior e inferior de los varones son mayores que los de las mujeres, la diferencia mostró significancia estadística para la prueba t de Student, a un $\mathrm{p}<0.01$, en todos los tipos de dientes, excepto en el caso de las segundas premolares superiores cuyo diámetro es algo mayor en las mujeres que en los varones, ver Tabla 2 y Tabla 3.

Las primeras premolares en el maxilar superior fueron mayores que las segundas premolares tanto en hombres como en mujeres, mientras que en el maxilar inferior la situación fue diferente, las segundas premolares fueron mayores que las primeras premolares para las mujeres y por lo contrario, las primeras premolares mayores que las segundas en los hombres. Situación similar a la encontrada por $\mathrm{Ku}$ bodera $^{8}$ en una población mexicana y diferente a lo encontrado por Singh ${ }^{5}$ en una población de niños del norte de India y Khan' en una población Bangladeshi, ver Tabla 4.

Los resultados de la influencia del sexo en el tamaño dental, muestran que los dien- 
tes de los hombres son consistentemente más grandes que los de las mujeres corroborándose datos reportados anteriormente referente a otras razas como caucásicos americanos $^{2,14,15}$ negros $^{13,14}$, japoneses ${ }^{18} \mathrm{y}$ chinos ${ }^{6}$.

En el maxilar superior las mayores diferencias entre géneros, estuvieron entre las primeras molares $(0.45 \mathrm{~mm})$ y segundas molares $(0.31 \mathrm{~mm})$, ver Fig. 1 , y en el maxilar inferior, entre los caninos (0.42) y las primeras molares (0.37), ver Fig.2. Resultados que confirman los hallazgos de otros investigadores ${ }^{2,3,10}$ en cuanto a los dientes que muestran las mayores diferencias entre géneros.

\section{Variabilidad de los diámetros mesiodis- tales}

Se calculó el coeficiente de variación (CV) para cada diente, los cuales se muestran en la Tabla 2 y Tabla 3, con el fin de identificar las piezas que presentan mayor variabilidad en sus mediciones, tanto en hombre como en mujeres. El coeficiente de variación se define como la relación de la desviación estándar con la media, expresando la desviación estándar como un porcentaje de la media.

Se encontró que la variabilidad fue mayor en hombres que en mujeres, 22 de 28 dientes, dato que se contrapone a lo hallado por Moorrees en una población caucásica y Kubodera en una población mexicana

Analizando la variabilidad por tipo de diente, se encontró que los mayores valores en el maxilar superior correspondieron a los incisivos laterales tanto en hombres (7.95\%) como en mujeres (7.80\%), y en el maxilar inferior fueron los incisivos centrales para los hombres $(7.41 \%) \mathrm{y}$ las segundas premolares para las mujeres (6.55\%), ver tabla 1 y tabla 2.

Las menores variabilidades fueron para las primeras molares superiores tanto en hombres $(5.03 \%)$ como en mujeres $(4.97 \%)$ y las primeras molares inferiores tanto en hombres $(5.26 \%)$ como en mujeres $(4.80 \%)$.

Estudios previos ${ }^{2,14}$ reportan también al IL como uno de los dientes más variables. Es de interés clínico la identificación de estas piezas, pues son los que tendrán mayor potencial para influir en el arco, en cuanto a la presencia de apińamiento, espaciamiento y/o discrepancia en el tamaño dental interarcadas.

\section{Conclusiones}

- En la muestra estudiada se encontró que en el maxilar superior la $1 \mathrm{M}$ presentó el mayor diámetro mesiodistal seguido en orden descendente por la 2M, IC, C, 1PM, 2PM, IL, en el maxilar inferior fue mayor la $1 \mathrm{M}$ seguido en orden descendente por la 2M, 2PM, 1PM, C, IL, C

- La diferencia de promedios entre dientes homólogos del lado derecho e izquierdo fue muy pequeńa en ambos géneros, no mostrando significancia estadística.

- Los diámetros coronarios mesiodistales del sexo masculino fueron significativamente mayores que los del femenino, excepto en el caso de la segunda premolar superior, las mayores diferencias se presentaron en la primera molar superior, canino inferior, primera molar inferior, segunda molar superior y segunda molar inferior

- Los dientes que presentaron mayor variabilidad de tamaño en el maxilar superior fueron los incisivos laterales en ambos géneros, y en el maxilar inferior, los incisivos centrales en los hombres y segundos premolares en las mujeres, los cuales se identifican como las piezas con mayor potencial para provocar alteraciones intra e interarcadas.

- Los promedios hallados en el presente estudio son mayores que los encontrados por otros autores, acentuándose este hecho a nivel de la $2 \mathrm{MS}$ que muestra ser mayor por más de $1 \mathrm{~mm}$.

\section{Referencias bibliográficas}

1. Moyers Robert E. Tratado de ortodoncia. México: Interamericana, 1960.

2. Moorrees CFA. Thomsen SO, Jensen E, Yen PK. Mesiodistal crown diameters of the deciduous and permanent teeth in individuals. J Dent Res 1957;36:39-47

3. Adeyemi TA, Isiekwe MC. Tooth size symmetry. A comparative analysis of tooth size among secondary school children. African Journal of Medicine and Medical Sciences 2004; 33(3):191-194.

4. Garino F, Garino GB. Comparison of dental arch measurement between stone and digital casts. World Journal of Orthodontics 2002;3:250-254.

5. Singh SP, Goyal A. Mesiodistal crown dimensions of the permanent dentition in North Indian children. J Indian Soc Pedod Prev Dent 2006;24:192-6

6. Ling JY, Wong RW. Tooth dimensions of southern chinese. Homo 2007;58(1):67-73

7. Austro, M.; Ostos, M.; García, C. \& Pérez, L. Análisis de la simetría del tamaño dentario mesiodistal de la misma muestra en dentición mixta y permanente. Estudio longitudinal. Av. Odontoestomatol. 2004;20(6):30513

8. Kubodera IT y cols. Dimensiones coronales mesiodistales en la dentición permanente de mexicanos. ADM. 2008;65(3):141-149.

9. Khan SH, Hassan GS, Rafique T, Hasan N, Hossain RS. Mesiodistal crown dimensions of permanent teeth in Bangladeshi population. BSMMU J. 2011;4(2):81-87.

10. Suazo GI, Cantín LM, López FB, Sandoval MC, Torres MS, Gajardo RP, Gajardo RM. Sexual dimorphism in mesiodistal and bucolingual tooth dimensions in chilean people. Int J Morphol. 2008;26(3). http://dx.doi-org/10.4067/S071795022008000300016

11. Perez FM, Fierro MC, Barboza P, Bravo LR, Torres ChF. Anchos mesiodistales en dentición temporal pura en niños y niñas de la ciudad de Concepcion, Chile. Int. J Odontostomat 2007;1(2):191-195.

12. Sanin C, Sabara BS. An análisis of permanent mesiodistal crown size. Am J Orthod 1971:59:488-500.

13. Richardson ER, Malhotra SK, Mesiodistal crown dimensions of the permanent dentition of American Negroes. Am J Orthod 1975;69:157-164

14. Kenee HJ. Mesiodistal Crown diameters of permanent teeth in male American Negroes. Am J Orthod 1979;76:95-99

15. Bishara SE, Jakobsen JR, Abdallah AF. Comparisons of mesio-distal and bucco-lingual crown dimensions of the permanent teeth in three populations from Egypt, Mexico and The United States. Am J Orhod \& Dentofac Orthoped 1989;96:416-22

16. Ghose LJ, Baghdady VS.Analysis of the dentition, mesiodistal crown diameters of permanent teeth. J Dent Res. 1979;58:1047-54

17. Lysell L, Myberg N. mesiodistal tooth size in the deciduous and permanent dentitions. Eur J Orthod. 1982;4:113-22.

18. Matsumoto M, Kuroda Y, Yoshida K, Hirata T, SakudaM. Harmony of tooth size between maxillary and mandibular dental arch in japanese. Nippon Kyosei Shika Gakkai Zashshi. 1971:30:52-55.

19. Guagliando MF. Tooth crown size differences between age groups; a possible new indicator of sress in skeletal samples. Am J Phys Anthrop. 1982;58:383-9 